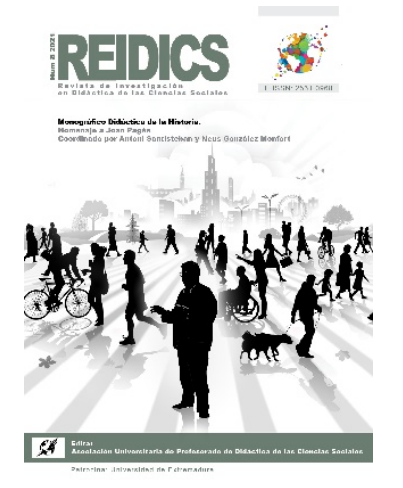

\title{
REIDICS
}

Revista de Investigación en

Núm. 8, 2021

Didáctica de las Ciencias

Sociales

Recibido 22 de enero de 2021

Aceptado 16 de febrero 2021

E-ISSN: 2531-0968

\section{Repensar la enseñanza de las Ciencias Sociales en tiempos de cambio}

\author{
Rethinking Social Science Teaching in Times of Change
}

Edda Sant

Manchester Metropolitan University

Email:e.sant@mmu.ac.uk

ORCID: https://orcid.org/0000-0002-7907-5907

DOI: https://doi.org/10.17398/2531-0968.08.23

\section{Resumen}

Este artículo tiene como objetivo reflexionar sobre la conceptualización de la enseñanza de las ciencias sociales. El artículo examina los fundamentos teóricos de la enseñanza de las ciencias sociales, particularmente en relación a la teoría liberal, marxista y la teoría crítica y hace un análisis de cómo los posicionamientos modernos han condicionado nuestra manera de entender la enseñanza y la didáctica de las ciencias sociales. A continuación, el artículo plantea la democracia radical como alternativa y señala algunas de las posibilidades que esta teoría abre para la enseñanza y el aprendizaje. El artículo, concebido como un tributo a Joan Pagès, pretende repensar algunas de las ideas del maestro a la luz de nuestros tiempos de cambio.

Palabras clave: Joan Pagès; democracia radical; enseñanza de las Ciencias Sociales; crisis de la modernidad; transposición didáctica.

\begin{abstract}
This article reflects about the way we conceptualise social studies (social science education). Firstly, the article examines the theoretical underpinnings of social science education, particularly in relation to liberal, Marxist and critical theory. Secondly, the paper analysis how modernity has conditioned the way we understand teaching and social science didactics. Thirdly, the article considers radical democracy as an alternative and examines some of the possibilities that this theory offer. This article is a tribute to Joan Pagès that aims to reconsider some of his ideas in our times of change.
\end{abstract}

Keywords: Joan Pagès; radical democracy; social studies; crisis of modernity; didactic transposition. 


\section{Introducción}

En los últimos años, las sociedades occidentales han experimentado un número de cambios políticos. Por un lado, hemos visto como los y las jóvenes, especialmente aquellos más marginados, son escépticos sobre las posibilidades de la política convencional (Sant \& Davies, 2018) y cuestionan si los sistemas en los que viven son genuinamente democráticos (Van de Velde, 2020). Al mismo tiempo, hemos visto el auge de partidos y movimientos de extrema-derecha, procesos de re-nacionalización y construcción de nuevos muros, constantes violaciones de los derechos humanos, desafíos a las instituciones democráticas por parte de líderes, etc. Muchos han atribuido estos cambios a una crisis de la modernidad y sus estructuras (e.g. Runciman, 2018; Santos, 2018). En su análisis de los textos de Gramsci, Babic (2020) explica que estamos en un momento de crisis orgánica y que estos cambios políticos son síntomas mórbidos que se viven durante los interregnos. Según Gramsci, en tiempos de interregno el sistema antiguo desaparece, pero el nuevo todavía no ha nacido. Los parámetros que utilizamos para el sistema antiguo ya no funcionan, pero no sabemos cuáles son los parámetros con los que nos vamos a encontrar en el futuro.

Joan Pagès decía que una de las preguntas clave que nos teníamos que formular antes de empezar a trabajar, pensar o enseñar es “¿para qué enseñamos ciencias sociales?”. Sobre el año 2002, escribió,

\footnotetext{
"Uno de los problemas más importantes de la enseñanza de las ciencias sociales y las humanidades es la escasa reflexión que se lleva a cabo sobre sus finalidades, sobre su incidencia en la configuración del pensamiento social del alumnado y su predisposición a la participación y la acción social. (...) El principio básico de la construcción del currículo es que el contenido no se incluye como un fin en sí mismo, sino que se selecciona como un medio para conseguir ciertos propósitos o finalidades." (2002a, p. 7).
}

Para Joan Pagès, como también para mí y para muchos otros, una de las finalidades, si no la finalidad, más esencial de la enseñanza de las ciencias sociales es lo que Santisteban (2011) define como la finalidad política o la posibilidad de facilitar que los y las jóvenes piensen alternativas, tomen decisiones y actúen en consecuencia. Gert Biesta (2011) denomina esta finalidad como subjetivación, el proceso mediante el cual la educación nos permite tomar decisiones de manera autónoma y generar procesos de cambio en el mundo. La finalidad política o de subjetivación, en este sentido, se refiere a lo que algunos denominarían "agencia" y otros, participación como ciudadanos democráticos.

En los últimos años Joan Pagès, más que nunca, reflexionó sobre el pasado y escribió sobre el futuro. Sus palabras en el último número de Perspectiva Escolar (marzo del 2020), revista en la que participó de manera decisiva durante años, fueron,

"Cal trobar la Perspectiva necessària per als temps que estem vivint, sense dogmatismes ni ortodòxies, amb el diàleg i amb l'esperança en l'enfortiment d'una societat i d'una escola cada vegada més democràtiques. Espero que Perspectiva trobi la perspectiva més escaient als temps que estem vivint." (Pagès, 2020, n.d.). 
Me arriesgaré a decir que con estas palabras Joan Pagès intuía que estos eran momentos de cambio, de crisis orgánica o de 'interregno'. En un email en marzo, me comentaba "parece que esto de la postmodernidad nos aguarda muchas sorpresas". Vivimos momentos en los que muchos no tenemos muy claro dónde vamos, y muchos otros ni tan siquiera donde estamos. Ver las noticias o leer el periódico cada día es una caja de sorpresas, muchas de ellas no muy agradables. Como él, yo también creo que es momento de tomar perspectiva. El contexto actual nos fuerza a tomar como punto de partida esta incertidumbre y a asumir que las bases modernas que estructuraban la manera en como entendíamos la democracia, la sociedad, y la enseñanza de las ciencias sociales pueden estar en crisis y no sabemos lo que vendrá después. En estas circunstancias, es importante repensar no solo para qué enseñamos ciencias sociales, sino como lo hacemos y porqué lo hacemos de esta manera. En este artículo me gustaría deconstruir algunas de las premisas que fundamentan nuestras prácticas y buscar alternativas a la luz de las teorías de la democracia radical. Para ello, empezaré el artículo analizando los fundamentos modernos de la enseñanza de las ciencias sociales, sus ventajas y sus problemáticas. A continuación, examinaré algunas de las ideas relacionadas con teorías de la democracia radical para intentar repensar la enseñanza de las ciencias sociales a la luz de estos tiempos. Mi objetivo es tomar perspectiva en estos tiempos de cambio y pensar como, reflexionando sobre nuestra memoria, afrontamos el presente y el futuro.

\section{Los fundamentos de la enseñanza de las ciencias sociales: liberalismo, marxismo y teoría crítica}

Tres tradiciones modernas han influenciado de manera determinante en la manera en como entendemos la enseñanza y la didáctica de las ciencias sociales: el liberalismo, el marxismo y la teoría crítica. La obra de liberales como Immanuel Kant es esencial para entender nuestras perspectivas actuales sobre la democracia, la educación, y la relación entre ambas (Biesta, 2007). La democracia actual tienes sus orígenes en lo que algunos llaman un matrimonio de conveniencia entre democracia y liberalismo, modelo que se ha constituido como hegemónico en muchos lugares del mundo, incluidos Europa y Latino América. En el modelo democrático-liberal, la democracia guarda una relación muy estrecha con la educación. Los ideólogos del modelo entendían que la educación era esencial para que los ciudadanos pudieran desarrollar todo su potencial y contribuir de manera decisiva en la construcción democrática (Barber, 1994). Conceptos como "educación para la democracia", o "para una ciudadanía democrática" son, en esta perspectiva, casi una tautología. La educación solo es si es educación para la democracia.

Esto es así puesto que, según Kant, existe una relación directa entre epistemología y ética, entre conocimiento y valores. Para Kant, la realidad social es objetiva: existe un sistema éticopolítico (la "ley moral") que es objetivamente y universalmente mejor que los otros (e.g. Corngold, 2011). Mediante la educación, podemos adquirir las habilidades de pensamiento crítico y racional que nos permitirán entender y valorar esta "ley moral" (Corngold, 2011). El término libertad, en Kant, se entiende como la posibilidad de que, una vez eliminados la ignorancia, los estereotipos y malentendidos, cada uno de nosotros puede guiarse por su propia racionalidad (Fraser-Burgess, 2012). En este sentido, los trabajos de Kant nos dejan como punto de partida una relación directa y unidireccional entre conocimientos, valores y libertad. 
Estos principios también sostienen muchas de las obras escritas por Karl Marx y de aquellos influenciados por él. En muchos de sus textos, Marx, al igual que Kant, defendió la universalidad de un sistema ético-político, en este caso, un sistema socialista (Walsh, 2008). En contraste con los liberales, Marx dio primacía a la economía sobre otros aspectos de nuestra vida social y puso el concepto de ideología en el centro de su análisis. Para Marx y sus discípulos, el capital mantenía su posición dominante mediante mecanismos de persuasión represivos e ideológicos que ocultaban la realidad tal y como esta era (Althusser, 1971). La educación podía ejercer el rol de mecanismo ideológico, pero también podía ejercer un papel mucho más emancipador. Michael Apple (2015), referencia a Antonio Gramsci, escribe,

"Cuando Gramsci (1971) argumentaba que una de las tareas de una educación contra-hegemónica y de un trabajador cultural comprometido no era descartar el "conocimiento de elite", sino reconstruir su forma y contenido para que fuera puesto al servicio de necesidades sociales genuinamente progresivas, otorgó una clave para otro rol que los intelectuales "orgánicos" y "públicos" pueden ejercer." (p. 34).

Las ideas marxistas llegaron a la didáctica de las ciencias sociales, principalmente a través de las disciplinas de origen (historiadores como Hobsbawm y geógrafos como Harvey) y también a través de la pedagogía crítica. Los trabajos de Hobsbawm, por ejemplo, acostumbran a utilizarse para examinar la construcción ideológica del nacionalismo en el sí de la enseñanza de las ciencias sociales (González, 2006; Sant, 2019). La escuela crítica parte de la tradición Freiriana y también de la actualización del trabajo de Marx llevada a cabo por la escuela de Frankfurt. Siguiendo a Freire (2000), la educación puede "deshumanizarnos": modelos tradicionales de enseñanza nos sumergen de manera más clara en estas ideologías opresivas, incluido el capitalismo, el patriarcado y el colonialismo. Pero la educación también puede "humanizarnos". Mediante la educación, podemos llegar a comprender las estructuras de poder que oprimen la vida de muchos y muchas, y condicionan la vida de todos, y cambiarlas. Educadores críticos como Giroux (2005) o McLaren y Garamillo (2008) entendieron que los y las docentes son agentes políticos que pueden intervenir en los procesos hegemónicos, utilizando estrategias para que los y las jóvenes comprendan los mecanismos de poder y actúen para cambiarlos. En una línea parecida, Pagès (2002b) escribió,

"Se concibe que la finalidad última de la enseñanza de la historia y las ciencias sociales ha de ser contrasocializadora, es decir, ha de preparar al alumnado para que construya sus propios conocimientos, se ubique en su mundo y esté preparado para intervenir en él de manera democrática" (p. 258).

Joan Pagès y muchos otros profesores y profesoras en didáctica de las ciencias sociales fueron pioneros en la defensa de una enseñanza de las ciencias sociales en particular, y una educación en general, que tuviera esta tarea contra-socializadora facilitando que el alumnado adquiriera los conocimientos necesarios para entender las estructuras de poder opresivas y cambiarlas. 


\section{La enseñanza de las ciencias sociales y el pensamiento moderno}

Desde mi punto de vista, la tradición liberal, la tradición marxista y la tradición crítica, se distinguen en su propósito, pero no tanto en sus suposiciones ontológicas y epistemológicas. Vestigios de estas tradiciones han quedado estampados en la manera en la que pensamos la enseñanza de las ciencias sociales. Estas tradiciones nos han dado, durante mucho tiempo, claridad y garantías para afrontar la enseñanza de las ciencias sociales y la formación del profesorado. Prats (2016), por ejemplo, escribió que la modernidad ha asegurado la supervivencia de las humanidades y las ciencias sociales en el sí de la universidad y de la escuela y también ha facilitado la construcción de un cuerpo de conocimiento que ha nutrido, expandido y, en de muchas maneras, mejorado la manera en la que muchos entendemos y experimentamos el mundo.

Sin embargo, las tradiciones modernas no son perfectas. Como los pensadores de las teorías decoloniales nos han demostrado el pensamiento moderno y su aplicación pueden ser muy oscuros y pueden, de manera intencional o no, reproducir estructuras opresivas de género, clase y "raza" (Mignolo, 2011; Santos, 2018). El pensamiento moderno, explica Boaventura de Sousa Santos (2006), toma como punto de partida dos principios o racionalidades. Primero, que existe una sociedad ideal y que poco a poco nos aceramos a este modelo. Esto es lo que Santos (2006) define como razón proléptica, en el "sentido de que ya sabemos cuál es el futuro: el progreso, el desarrollo de lo que tenemos" (p. 21). Segundo, que existen unos mecanismos objetivos para construir conocimiento absoluto. Esto es lo que Santos (2006) denomina la razón metonímica, una razón que "contrae el presente porque deja por fuera mucha realidad, mucha experiencia, y al dejarlas afuera, al tornarlas invisibles, desperdicia la experiencia" (p. 21).

Joan Pagès, consciente de estos problemas, encabezó un nuevo movimiento que reclamaba un cambio en los contenidos de ciencias sociales para visibilizar aquellos y aquellas que no aparecían en la historia. Los ya numerosos trabajos sobre los "invisibles" demostraron la poca presencia de mujeres, niños, y niñas, y minorías étnicas entre otros en estos contenidos (e.g. Pagès Blanch \& Sant, 2012; Pagés Blanch, Villalón Gálvez \& Zamorano Varga, 2017; Pinochet Pinochet \& Pagès Blanch, 2016; Marolla-Gajardo, 2019). Pagès explicaba que, si los jóvenes y las jóvenes no se veían reflejados en la historia enseñada, no se sentirían protagonistas de su presente y constructores de su futuro. También denunciaba que los contenidos de historia y ciencias sociales daban una visión muy partidaria y exclusiva de lo que era ser humano. Más recientemente, Massip Sabater, Castellví Mata y Pagès Blanch (2020) demostraron que, en los últimos 25 años, nuevas tendencias historiográficas han democratizado y des-objetivizado la historia, pero el currículo de historia (y de ciencias sociales) no se ha adaptado a estos cambios, continuando enseñando una historia positivista, unidimensional, discriminatoria y alejada de la vida de los y las jóvenes. En este artículo, los autores consideraban una posible democratización de la enseñanza de las ciencias sociales y para ello recomendaban desarrollar dos de las líneas de trabajo de Fontana:

"En primer lugar, a través de una forma que abandonara la narrativa y optase por un tipo de relato polifónico que trabajase con historias de vida y "que escogiese el número suficiente de las voces altas y bajas, grandes y pequeñas, de la historia para articularlas en un coro más significativo" (Fontana, 2013, p. 192); por otro lado, a partir de acontecimientos concretos y no de soluciones preestablecidas; y finalmente, 
abandonando la linealidad "que nos ayudará no solo a superar el eurocentrismo, sino también el determinismo (p. 195).” (p. 189)

Sin duda sus sugerencias abren la posibilidad a una democratización de los contenidos que nos ayudaría a superar algunos de los déficits que la razón proléptica y la razón metonímica ha dejado en las aulas y en nuestra sociedad. Sin embargo, y aunque totalmente de acuerdo con estos autores, considero que la razón proléptica y la razón metonímica operan a un nivel mucho más subyacente en la manera en como entendemos la enseñanza de las ciencias sociales.

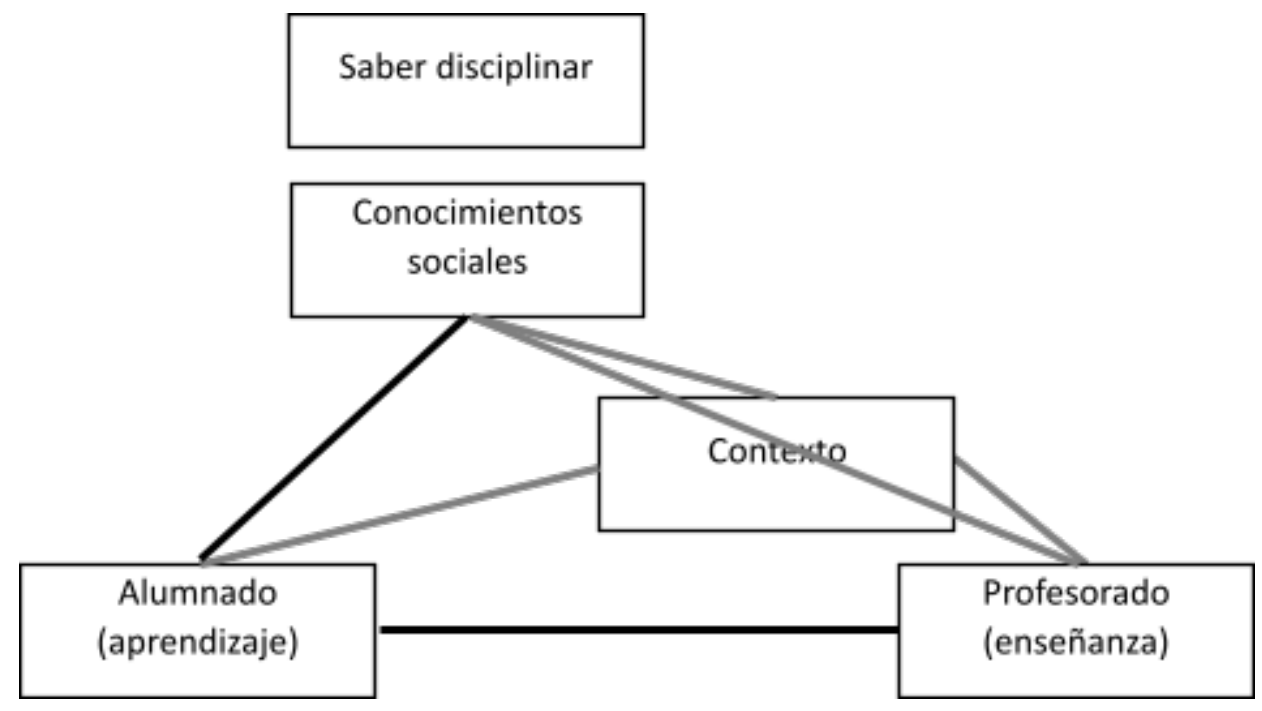

Figura 1. Simplificación del modelo tradicional de la pirámide didáctica y la transposición didáctica

\section{Fuente: Elaboración propia}

Desde mi punto de vista, la enseñanza de las ciencias sociales (y seguramente también la enseñanza de las otras disciplinas) acostumbra a partir de una lógica racionalista y/o objetivista que opera al nivel de la razón metonímica. Consideren, por ejemplo, el modelo de la pirámide o triángulo didáctico que he intentado reproducir y simplificar en la figura 1. Este modelo, en sus distintas variaciones, ha articulado la comprensión, interpretación y formación de muchos de aquellos que nos dedicamos a la enseñanza y/o la didáctica de las ciencias sociales (e.g. Santisteban y Pagès, 2011). En el triángulo, aparecen siempre tres elementos clave: "el profesor como experto, el alumno como aprendiz y el saber que justifica la relación profesor-alumno y que responde a unas finalidades educativas y sociales" (Pagès, 2011, p. 215). Obviamente, el triángulo es una simplificación explícitamente diseñada con el propósito de señalar los aspectos indispensables que la didáctica tiene que considerar, y en este sentido, cumple su función. Sin embargo, desde mi punto de vista, el triángulo ilustra como la epistemología racionalista y objetivista ha influenciado la enseñanza y la didáctica de las ciencias sociales de manera clara pero no siempre obvia. Los conocimientos o contenidos concretos aparecen desvinculados de los sujetos que los crearon, aquellos y aquellas que los enseñan y aquellos y aquellas que los aprenden. Tendemos a considerar los contenidos como una entidad en sí, objetiva y separada de nuestras subjetividades. 
Esta conceptualización racionalista y/o objetivista no deja mucho lugar a otras maneras de experimentar el mundo y nuestro lugar en él. Los y las jóvenes aprenden que solamente mediante la razón podemos llegar a conocer, y únicamente la razón será valorada en el sí del contexto educativo. La transposición didáctica nos dice que el conocimiento válido en las aulas de ciencias sociales es el saber sabio que solo se transforma en saber escolar para ser digerido por el alumnado. La concepción Kantiana que adquirir conocimientos nos hace mejores persones es, en el mejor de los casos una perspectiva naíf y en el peor de los casos una perspectiva elitista y discriminatoria. Como Walter Mignolo (2011) comenta, la modernidad tiene dos caras y es difícil, si no imposible, mirarle la cara la ilustración e ignorar los procesos coloniales. Lo que es el "conocimiento racional" solo puede constituirse mediante las exclusiones de lo que no lo es. Construcciones racionalistas y objetivistas de la realidad son por naturaleza socialmente excluyentes y lo mismo sucede en las aulas. De esta manera, las experiencias de muchos y muchas (particularmente aquellos y aquellas jóvenes que ya están marginalizados de por sí) son silenciadas, excluidas o considerados no valiosas (Gibson, 2020). En las aulas muchas veces se oyen las voces lejanas e interpretadas de los académicos, pero no tanto las de los y las que están allí.

La enseñanza de las ciencias sociales también acostumbra a partir una lógica curricular que reproduce la razón proléptica. Sin lugar a duda, como bien identificaron Massip, Castellví y Pagès (2020), esto sucede de manera explícita en las construcciones cronológicas del Curriculum de historia. Pero la imprenta de la razón proléptica va más allá. Aprender ciencias sociales se presenta como un proceso acumulativo y lineal. Se entiende que los niños y las niñas progresan en su adquisición de conocimientos y que la enseñanza de las ciencias sociales les prepara para ejercer la ciudadanía democrática. Pagès (2011) explicaba es "el experto, el profesor, que ha de conseguir acercar el saber al aprendiz para que de alguna manera pueda utilizarlo para seguir aprendiendo y para ubicarse en su mundo" (p. 215) (ver figura 2).

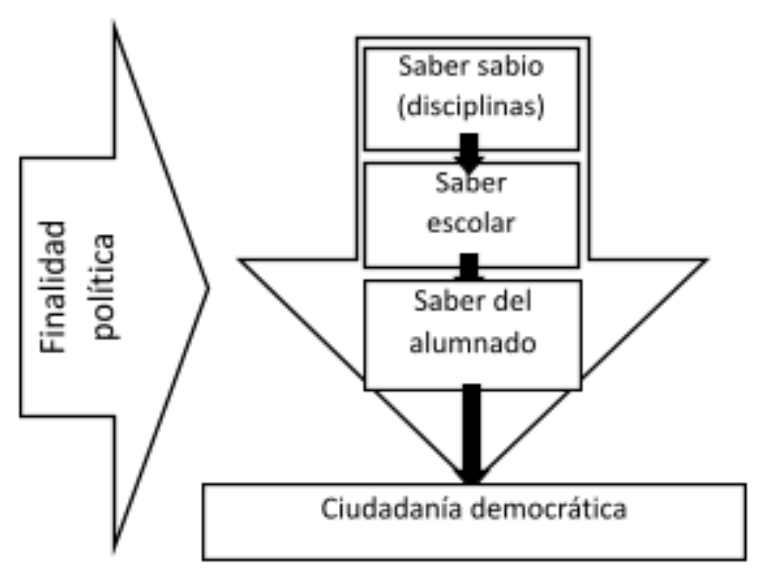

Figura 2. Simplificación de la transposición didáctica en relación a las finalidades

Fuente: Elaboración propia 
Esta enseñanza de las ciencias sociales que tiene como objetivo la educación para la democracia tiene muchas virtudes. Pero también tiene defectos principalmente relacionados con lo que Santos denomina la razón proléptica. Primero, esta concepción corre el riesgo de resultar frustrante si no engañosa. Tenemos que admitir que el concepto de "finalidad" implica destino y linealidad, pero la enseñanza de las ciencias sociales nunca ha sido una "ciencia exacta" que se pueda predecir y aunque sepamos "donde queremos ir", no siempre llegamos a ese "fin". La concepción Freiriana que, los oprimidos cuando en diálogo con sus maestros, llegarán a la conclusión que la solidaridad entre oprimidos es esencial, se ve cuestionada cuando observamos acontecimientos recientes. Por ejemplo, las últimas investigaciones señalan que proyectos de formación del pensamiento crítico dirigidos a grupos de extrema derecha pueden tener resultados contra-productivos (Miller-Idriss \& Pilkington, 2020). La también concepción Freiriana, altamente relevante para la pedagogía crítica, que la educación y el pensamiento crítico llevan a la acción social, es cuestionable (Castellví Mata, 2019). En mi propio trabajo, he comprobado como los y las jóvenes que demuestra una comprensión crítica de nuestra realidad pueden llegar a ser muy cínicos y apáticos (Sant \& Davies, 2018).

Segundo, esta construcción lineal de la enseñanza es determinista en relación a lo que los y las jóvenes deberían ser. Tenemos en mente, Biesta (2007) explica, una visión ideal de lo que ser humano debería ser, e intentamos amollar las nuevas generaciones a estos modelos. Los y las jóvenes se definen como "todavía-no" ciudadanos o como ciudadanos y ciudadanas de segunda que no son todavía "competentes" (Biesta \& Lawy, 2006). El rol de los y los docentes es el de acompañar a los y las jóvenes para que estos se conviertan en ciudadanos de bien. Limitamos (o intentamos limitar) las opciones de futuro a los y las jóvenes del presente.

\section{La democracia radical como alternativa}

Los autores decoloniales han ofrecido, desde mi punto de vista, una crítica muy convincente de la modernidad. Sin embargo, como el mismo Santos (2018) indica, una de las opciones posibles es intentar apropiarnos, de manera contrahegemónica de las ideas, conceptos y prácticas que la modernidad nos ha dejado. En los últimos años, mi trabajo se ha centrado en repensar la enseñanza de las ciencias sociales a la luz de las teorías de la democracia radical. Creo que estas teorías nos ofrecen algunas pistas sobre cómo podemos replantearnos la enseñanza de unas ciencias sociales que nos permitan aliviar los efectos más devastadores de la razón proléptica y la razón metonímica, o, como mínimo, reconocer nuestra complicidad en estos procesos.

Las teorías de la democracia radical utilizan el término radical de manera diferente a lo que estamos acostumbrados en el sí de la didáctica. Radical, en didáctica y enseñanza de las ciencias sociales, generalmente se entiende en relación a las obras de autores críticos como Henry Giroux (2003) o Wayne E. Ross (Ross y Vinson, 2012) que intentan llevar los principios de la teoría crítica a sus límites para generar un cambio social radical. Sin embargo, el término radical, en el sí de las teorías de la democracia radical, tiene connotaciones diferentes. Para explicarlas es útil referirnos a la construcción etimológica del término "radical": raíz. La democracia radical tiene como objetivo cuestionar "las raíces" de nuestras prácticas sociales. Sarah Amsler (2016) define la democracia radical como el grupo de proyectos que tienen en común el compromiso político de, 
1. "Mantener un escepticismo anarquíco hacia la verdad y el poder,"

2. "Facilitar el trabajo práctico que estos compromisos requieren", y

3. "Liberar posibilidades de la imposición de la "falsa necesidad" (p. 73).

Los diferentes movimientos académicos, sociales y políticos relacionados con la democracia radical se han nutrido del trabajo de diferentes filósofos y politólogos entre ellos Jaques Rancière, Chantal Mouffe y Ernesto Laclau. Estos autores, generalmente definidos como post-marxistas, alteran las "raíces" de tres de los principios generalmente asumidos por autores liberales y críticos.

En primer lugar, estos autores entienden la realidad social como un sistema de diferencias en el que ideas y conceptos se construyen mediante procesos discursivos hegemónicos. Siguiendo las lógicas del pensamiento post-estructuralista, estos autores asumen que no existe separación entre los objetos y los sujetos de estudio, entre lo que generalmente se denomina razón y emoción. Laclau (2007b) explica que si los sujetos son históricamente finitos, su conocimiento también lo es. A ello, Mouffe (2018) añade,

"Las cosas siempre podrían haber sido distintas y todos los sistemas se crean mediante la exclusión de otras posibilidades. Siempre son la expresión de una configuración particular de poder sin ninguna base racional. Las cosas que nos parecen muy naturales hoy no lo son (...). Todos los sistemas son susceptibles al cambio mediante prácticas contra-hegemónicas" (p. 88).

En este sentido, para los demócratas radicales, como también para otros como Foucault (1980), no existe separación entre conocimiento y poder. Toda forma de conocimiento se construye mediante mecanismos de poder a través de los cuales únicamente unos pocos tienen la posibilidad de definir lo que es y lo que no es conocimiento, lo que es y lo que no es racional (ver también Ortega Sánchez \& Pagès Blanch, 2019). Radicalizar la democracia, en este sentido, significa politizar los espacios sociales y los temas de debate, incluso aquellos que dábamos "por supuesto" dado que estaban probados por la historiografía, la geografía o ninguna otra disciplina de referencia. Radicalizar la democracia significa aquí democratizar el conocimiento, no solo haciendo que las disciplinas lleguen a todos, pero que todos lleguen a participar en su construcción.

En segundo lugar, estos autores cuestionan la expectativa ontológica que existe un único sistema ético-político preferible y que aquellos que tienen los conocimientos más elaborados están en una mejor posición para definir este sistema preferible. De acuerdo con Rancière, el problema de las posiciones marxistas ortodoxas es que toman el principio de igualdad como destino o finalidad de toda actividad política, y en hacerlo imponen una visión elitista y única a todos incluso a aquellos a los que pretenden representar. Según Rancière, debemos tomar el concepto de igualdad como punto de partida. Todos los seres humanos tienen la habilidad de "hablar" y ocuparse de asuntos comunes (Barbour, 2010), o lo que Rancière denomina como la igualdad de inteligencias. Todos podemos contribuir y participar en los asuntos de nuestra comunidad.

Esta reconceptualización del término igualdad tiene efectos importantes para el acto educativo. Rancière destaca que, en la educación convencional, el "profesor es por definición el que sabe hacia dónde conducir al ignorante en la progresión de los saberes que reproduce la 
relación desigual" (en Giuliano y Cantarelli, 2016, p. 616). En contraste, él propone que reimaginamos el profesor como un maestro ignorante o como "aquel que nos pone en movimiento y nos hace aprender lo que él mismo no sabe, en la medida en que su autoridad nos conduce a un punto que ni él ni nosotros conocemos con anterioridad" (2016, p. 616). En este sentido, Rancière defiende separar la condición de sabio de la de maestro. En líneas parecidas, Laclau (1990) examina el rol de los intelectuales. Para el autor, los intelectuales no deben estar a la vanguardia de la lucha política y social, como dijo Gramsci, sino favorecer la disolución de su propia casta, facilitando que haya menos grandes intelectuales, y más intelectuales. Radicalizar la democracia significa aquí tomar como punto de partida que todos los seres humanos son libres e iguales en su rol como actores políticos.

En tercer lugar, Laclau y Mouffe (2001) defienden que si la democracia es posible es precisamente porque es abierta. En uno de sus textos, Laclau (2007) imagina una sociedad acabada, sin conflicto y disenso, y concluye que este mundo sería una sociedad distópica:

\footnotetext{
"Sería una sociedad absolutamente unidimensional, en la que el $100 \%$ de la población estaría de acuerdo con cada una de las reformas, o una sociedad en la que las decisiones serían tomada por un equipo de ingenieros sociales sin el apoyo de la población" (p. 114).
}

Desde esta perspectiva, y en contra de las propuestas de Kant y también Marx, el concepto libertad es opuesto al concepto de determinación. Imaginar una sociedad acabada es lo mismo que imaginar una sociedad cerrada, y, en consecuencia, no democrática. Laclau y Mouffe (2001) consideran que la democracia requiere de un equilibrio frágil entre aspiración y pragmática, entre lo que deseamos y lo que hacemos. Por ejemplo, intentamos hacer leyes que garanticen la igualdad para todos, pero estas leyes siempre fallan en algún u otro aspecto y esto nos permite explorar nuevos caminos para definir y repensar la igualdad y su práctica. Es precisamente esta brecha entre aspiración e intento fallido lo que abre nuevas posibilidades y nos permite cambiar, adaptarnos e incorporar los deseos de las nuevas generaciones al proyecto democrático. Radicalizar la democracia, de esta manera, también quiere decir reconocer que la incertidumbre, aunque asuste, puede abrir nuevas posibilidades para el futuro.

\section{Repensar la enseñanza de las ciencias sociales a través de la democracia radical}

$\mathrm{Si}$, en resumen, entendemos la democracia radical en base a estos tres principios: 1) todos los aspectos de la vida social, incluso el conocimiento, es político; 2) todos los seres humanos son capaces de participar en la vida política; 3 ) la democracia es un proyecto abierto, ¿cómo podemos repensar una enseñanza de las ciencias sociales cuya función sea la educación política y que reconozca e intenta aliviar los efectos de las razones prolépticas y metonímicas?

Déjenme retomar el concepto de transposición didáctica para ilustrar la propuesta. En páginas anteriores, la figura 2 ejemplificó la manera en como la transposición didáctica generalmente se entiende; la figura 3 ejemplifica aquí la manera en como creo que podemos repensar la enseñanza de las ciencias sociales. En este modelo, entendemos que todo conocimiento es parcial y está vinculado a aquellos que lo construyeron/experimentaron. Todos 
los saberes, en este sentido, son saberes "sabios". La lógica curricular y la lógica de la transposición didáctica tradicional funcionan de arriba-abajo. Generalmente, las disciplinas generan conocimientos que son luego filtrados por aquellos que diseñan el currículum (y muy frecuentemente los libros de texto) y luego adaptados por los y las docentes que lo transforman en saber escolar. Los y las docentes seguimos este proceso de "traducción" consciente o inconscientemente. Esta es una traducción unidireccional. Mi propuesta es repensar la transposición didáctica como un proceso bidireccional. No se trata de modificar el rol de la enseñanza ni el trabajo de los docentes, sino de ampliarlo. El rol de los y las docentes es, no únicamente facilitar que los conocimientos académicos lleguen al alumnado, sino que los conocimientos y experiencias del alumnado lleguen fuera de las aulas. El rol del profesorado aquí no es únicamente el rol del sabio, sino el del maestro o la maestra que pone en movimiento las experiencias históricas, geográficas y políticas del alumnado sin saber de antemano donde estas experiencias nos van a llevar. Es también el rol del intelectual orgánico Laclaudiano que no pretende estar, en su condición de maestro o maestra, a la vanguardia de la lucha política sino a la retaguardia.

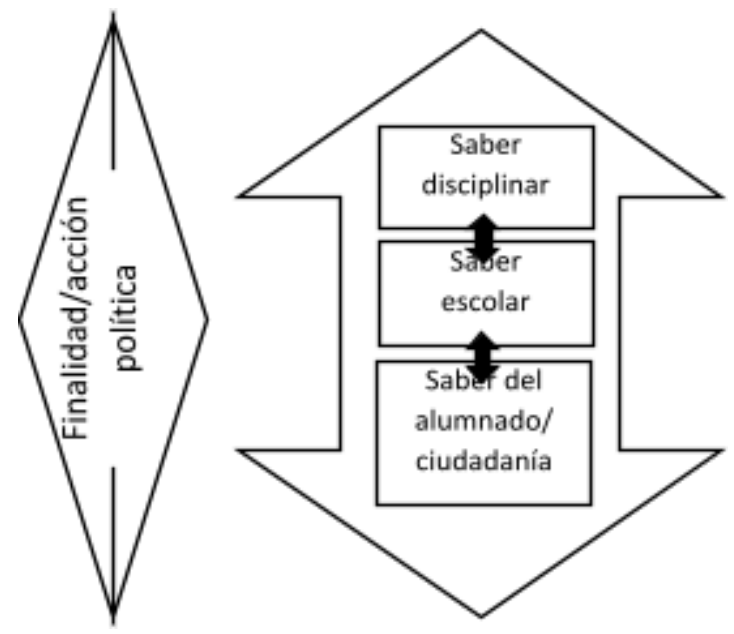

Figura 3. Simplificación de la transposición didáctica bidireccional en relación a las finalidades

\section{Fuente: Elaboración propia}

Pensar la enseñanza de las ciencias sociales en relación a esta transposición didáctica bidireccional nos ayuda a entender otros modelos de enseñanza que faciliten la toma de decisiones de manera autónoma y la acción social del alumnado sin caer en los dilemas asociados con la razón proléptica y la razón metonímica. Como he identificado al principio de este artículo, para mí como para muchos, el propósito de la enseñanza de las ciencias sociales es facilitar que el alumnado sea agentico: que pueda tomar decisiones de manera autónoma y generar procesos de cambio en el mundo. Creo que los y las docentes y didactas de ciencias sociales tenemos mucho que aportar, estamos en una posición inmejorable para entender cómo han funcionado y funcionan los procesos de cambio en el mundo, y las oportunidades y los retos que los y las jóvenes se pueden encontrar si intentar ser estos agentes de cambio. Sin embargo, no concibo estas ideas únicamente como una finalidad a largo plazo sino como un propósito del día a día. Los y las jóvenes 
son los y las ciudadanas del futuro pero también del presente. Hemos invertido mucho tiempo pensando en cómo enseñar historia a las nuevas generaciones para que estos hagan política, quizá es hora de pensar cómo ayudarles a hacer política para que hagan historia.

\section{A modo de conclusión}

Acontecimientos políticos recientes han demostrado que las estructuras modernas están en crisis y que vivimos en tiempos de cambio. Desde mi punto de vista, estos acontecimientos van más allá de lo que sucede en las aulas de ciencias sociales y de lo que los y las docentes y didactas pueden controlar o influenciar. Sin embargo, estos acontecimientos parecen indicar que estamos llegando al fin de una era y que todavía no sabemos lo que va a venir después. En esta situación de cambio y transición creo que es indispensable tomar perspectiva y pensar como la enseñanza de las ciencias sociales puede adaptarse a estos momentos de incertidumbre.

En este artículo, he intentado emplear teorías de la democracia radical para añadir posibilidades en la manera en como entendemos y practicamos la enseñanza de las ciencias sociales. Si seguimos los principios de la democracia radical, podemos entender que todos los aspectos de nuestra realidad (incluso el conocimiento) son políticos, todos los humanos son capaces de participar en la vida política y construir día a día nuevas formas de democracia. Esta reconceptualización nos ayuda a imaginar un modelo suplementario para la enseñanza de las ciencias sociales. Modelo, en el que, el rol de los y las docentes no es tan solo educar a los y las jóvenes en los conocimientos establecidos, pero ayudar a estos y estas jóvenes a participar en la construcción política de nuevos conocimientos. Este modelo se puede ilustrar en la forma de una transposición didáctica bidireccional que pretende no únicamente acercar la realidad social al alumnado sino también favorecer que el alumnado participe directamente en la construcción de esta realidad.

Reconozco que este no es un artículo habitual. En sus líneas mezcla discusiones más académicas con encuentros y menciones más personales relativos a la vida y obra de (o a mis encuentros con) Joan. Para mí, no podía ser de otra manera. Cuando Antoni Santisteban y Neus González me pidieron que escribiera este artículo me pusieron delante una pregunta imposible. ¿Escribir sobre lo académico, o escribir sobre mi maestro? ¿Cómo podría escribir sobre el uno, y no sobre el otro? ¿En un homenaje a Joan, cómo podría escribir sobre lo que pienso y no sobre lo que siento? La consecuencia de esta pregunta imposible es un artículo ecléctico que ilustra las dificultades de escribir (o la artificialidad del pensamiento moderno aun imperante) en el mundo académico.

También escribo estas líneas finales consciente que mi análisis y preguntas en este artículo pueden ser interpretadas como un reto a la enseñanza de las ciencias sociales o a la finalidad política de estas. Me gustaría, para finalizar, clarificar que mi intención es totalmente distinta. Escribo este artículo como un tributo a Joan Pagès, y mi objetivo no es otro que sugerir algunas posibilidades, que a la vista de lo que ya sabemos y en el contexto de incertidumbre de nuestros tiempos, nos permitan continuar trabajando para educar "ciudadanos comprometidos que sepan lo que están haciendo, y lo hagan junto con los demás, para construir un mundo más justo, más solidario y mucho más igualitario que el que tenemos actualmente" (Pagès Blanch, 2009, p. 152). 
En su artículo en Perspectiva Escolar Joan explicaba "Hoy, con este número, se pone fin a una etapa. Y empieza otra en la que yo no estaré. Es tiempo de dar paso a las nuevas generaciones para que construyan la Perspectiva de lo que queda del siglo XXI" y luego concluía "[y]o seguiré pensando, con nostalgia de un pasado vivido intensamente, como puedo ayudar en este nuevo camino des de mi perspectiva" (2020, n.d.). El reto ahora, para muchos de nosotros, es caminar sin su presencia, pero siempre, con su ayuda.

\section{Referencias bibliográficas}

Althusser, L. (1971). Ideology and the State. Lenin and philosophy and other essays, 2.

Amsler, S. S. (2016). The education of radical democracy. London: Routledge.

Apple, M. W. (2015). Conocimiento, poder y educación: sobre ser un académico/activista. Entramados: educación y sociedad, (2), 29-39.

Babic, M. (2020). Let's talk about the interregnum: Gramsci and the crisis of the liberal world order. International Affairs, 96(3), 767-786.

Barber, B. R. (1994). An aristocracy of everyone. Oxford, England: Oxford University Press

Barbour, C. A. (2010). Militants of truth, communities of equality: Badiou and the ignorant schoolmaster. Educational Philosophy and Theory, 42, 251-263

Biesta, G. (2007). Education and the democratic person: Towards a political understanding of democratic education. Teachers college record, 109(3), 740-769.

Biesta, G. J. J. (2011). Learning democracy in school and society: Education, lifelong learning, and the politics of citizenship. Dordrecht: Springer.

Biesta, G., \& Lawy, R. (2006). From teaching citizenship to learning democracy: overcoming individualism in research, policy and practice. Cambridge journal of education, 36(1), 63-79.

Castellví Mata, J. (2019). Literacitat crítica digitial en els estudis socials: estudis de cas en Educació Primària. Tesis Doctoral.

Corngold, J. (2011). Misplaced priorities: Gutmann's democratic theory, children's autonomy, and sex education policy. Studies in Philosophy and Education, 30, 67-84.

Foucault, M. (1980). Power/knowledge: Selected interviews and other writings, 1972-1977. Vintage.

Fraser-Burgess, S. (2012). Group identity, deliberative democracy and diversity in education. Educational Philosophy and Theory, 44, 480-499.

Freire, P. (2000). Pedagogy of the oppressed (30th anniversary ed.). London, England: Bloomsbury.

Gibson, M. (2020). From deliberation to counter-narration: Toward a critical pedagogy for democratic citizenship. Theory \& Research in Social Education, 1-24.

Giroux, H. A. (2003). Public pedagogy and the politics of resistance: Notes on a critical theory of educational struggle. Educational philosophy and theory, 35(1), 5-16.

Giroux, H. A. (2005). Estudios culturales, pedagogía crítica y democracia radical. Madrid: popular

Giuliano, F., \& Cantarelli, M. N. (2016). La Igualdad en la Revuelta Educativa: una conversación con Jacques Rancière. Educação \& Realidade, 41(2), 613-627.

Gonzalez Valencia, G. A. G. (2013). El profesorado en formación y las finalidades de la enseñanza de las ciencias sociales. Uni-pluriversidad, 13(2), 24-34. 
González, M. P. (2006). Conciencia histórica y enseñanza de la historia: una mirada desde los libros de texto. Enseñanza de las ciencias sociales, (5), 21-30.

Laclau, E. \& Mouffe, C. (2001). Hegemony and socialist strategy: Towards a radical democratic politics (2nd edn). London: Verso.

Laclau, E. (1990). New Reflections on the Revolution of Our Time. London: Verso

Laclau, E. (2007). Emancipation(s). London: Verso.

Marolla-Gajardo, J. (2019). La ausencia y la discriminación de las mujeres en la formación del profesorado de historia y ciencias sociales. Revista Electrónica Educare, 23(1), 137-157.

Massip Sabater, M., Castellvi Mata, J., \& Pagès Blanch, J. (2020). La historia de las personas: reflexiones desde la historiografía y de la didáctica de las ciencias sociales durante los últimos 25 años. Panta Rei. Revista digital de ciencia y didáctica de la Historia, 14(2), 167196.

McLaren, P., \& Jaramillo, N. (2008). Globalitzacions alternatives: cap a un estudi crític de la globalització: Neoliberalisme, globalització capitalista i la crisi de l'esquerra educativa. Rizoma freireano, (1), 1.

Mignolo, W. (2011). The darker side of western modernity: Global futures, decolonial options. Duke University Press.

Miller-Idriss, C., \& Pilkington, H. (Eds.). (2020). Gender and the Radical and Extreme Right: Mechanisms of Transmission and the Role of Educational Interventions. Routledge.

Navarro Medina, E. (2018). Un ciclo de mejora docente para la formación de maestros y maestras. En Didáctica de las Ciencias Sociales. Jornadas de Formación e Innovación Docente del Profesorado, 1, (pp. 898-921). Universidad de Sevilla.

Ortega Sánchez, D. \& Pagès Blanch, J. (2019). Las emociones y los sentimientos en la enseñanza de la historia y de las ciencias sociales. En Maria Joao Hortas, Alfredo Dias, Nicolás de-AlbaFernández (coord.) Enseñar y aprender didáctica de las ciencias sociales: la formación del profesorado desde una perspectiva sociocrítica (pp. 118 - 127). Ediciones Escola Superior de Educação.

Pagès Blanch, J. (2011). Conversatorio entre Joan Pagès y docentes de la escuela de Ciencias Sociales de la Universidad Pedagógica y Tecnológica de Colombia. Revista Historia y Memoria, (3), 203-226.

Pagès Blanch, J., \& Sant E. (2012). Las mujeres en la enseñanza de la Historia: ¿hasta cuándo serán invisibles? Cadernos de Pesquisa do CDHIS, 25(1), 91-117.

Pagès Blanch, J., Villalón Gálvez, G., \& Zamorano Vargas, A. (2017). Enseñanza de la historia y diversidad étnica: los casos chileno y español. Educação \& Realidade, 42(1), 161-182.

Pagès, J. (2002a). Preguntas y problemas del currículo de ciencias sociales en la Enseñanza Obligatoria. Preparación para la acción. Benejam, P. (coord.): Enseñanza de las Ciencias Sociales. Madrid: Santillana Educación.

Pagès, J. (2002b). Aprender a enseñar historia y ciencias sociales: el currículo y la didáctica de las ciencias sociales. Pensamiento educativo, 30(1), 255-269.

Pagès, J. (2009). Enseñar y aprender ciencias sociales en el siglo XXI: reflexiones casi al final de una década. In II Congreso Internacional. Investigación en Educación, Pedagogía y Formación 
Docente (pp. 140-154). Medellín. Universidad pedagógica nacional, Universidad de Antioquia, Corporación interuniversitaria de servicios.

Pagès, J. (2020). Mirada. Una mirada... una història i un futur amb perspectiva per a Perspectiva Escolar. Perspectiva Escolar, 410, https://www.rosasensat.org/revista/poesia-per-totnum-410/mirada-una-mirada-una-historia-i-un-futur-amb-perspectiva-per-aperspectiva-escolar/

Pinochet Pinochet, S., \& Pagès Blanch, J. (2016). ¿Cuál es el protagonismo de los niños, las niñas y los jóvenes en el currículo de Historia, Geografía y Ciencias Sociales de Chile?: El impacto del currículo en las ideas de los niños y de los jóvenes sobre su protagonismo en la Historia. Praxis Educativa, 11(2), 374-393.

Prats, J. (2016). Combates por la Historia en la educación. Enseñanza de las ciencias sociales: revista de investigación, (15), 145-153.

Ross, W. E., \& Vinson, K. D. (2012). La educación para una ciudadanía peligrosa. Enseñanza de las ciencias sociales: revista de investigación, 11, 73-86.

Runciman, D. (2018). How democracy ends. London: Profile Books.

Sandahl, J. (2015). Social studies as socialisation, qualification and subjectification. In European Consortium for Political Research, General Conference, Montreal, Canada, 26-29 August 2015.

Sant, E. (2019). National myths and democratic history education: secondary students' discursive construction of Catalan nationhood. Pedagogy, Culture \& Society, 1-19, https://www.tandfonline.com/doi/full/10.1080/14681366.2019.1703793

Sant, E., \& Davies, I. (2018). Promoting participation at a time of social and political turmoil: what is the impact of children's and young people's city councils? Cambridge Journal of Education, 48(3), 371-387.

Santisteban, A. \& Pagès, J. (2011). Didáctica del Conocimiento del Medio Social y Cultural en la Educación Primaria. Madrid: Síntesis.

Santisteban, A. (2011). Las finalidades en la enseñanza de las Ciencias sociales. En Santisteban, A. \& Pagès, J. (2011). Didáctica del Conocimiento del Medio Social y Cultural en la Educación Primaria (pp. 63-83). Madrid: Síntesis

Santos, B. de Sousa (2006). Renovar la teoría crítica y reinventar la emancipación social. Clacso.

Santos, B. de Sousa (2018). The end of the cognitive empire: The coming of age of epistemologies of the South. Duke University Press.

Van de Velde, C. (2020). A global student anger? A comparative analysis of student movements in Chile (2011), Quebec (2012), and Hong-Kong (2014). Compare: A Journal of Comparative and International Education, 1-19.

Walsh, J. (2008). The critical role of discourse in education for democracy. Journal for Critical Education Policy Studies, 6(2), 54-76. 